\title{
Effect of a distributed delay on relative stability of diffusely coupled systems, with application to synchronized equilibria
}

\author{
I.-C. Morărescu ${ }^{1 *}$, W. Michiels ${ }^{2}$, M. Jungers ${ }^{1}$ \\ ${ }^{1}$ Université de Lorraine, CRAN, UMR 7039 and CNRS, CRAN, UMR 7039, 2 av. Forêt de Haye, Vandouvre-lès-Nancy, \\ France Constantin. Morarescu@univ-lorraine.fr, Marc. Jungers@univ-lorraine.fr \\ ${ }^{2}$ Department of Computer Science, Katholieke Universiteit Leuven, Celestijnenlaan 200A, 3001 Heverlee, Belgium \\ Wim.Michiels@cs.kuleuven.be
}

\begin{abstract}
SUMMARY
We perform a linear stability analysis of synchronized equilibria in networks of diffusely coupled oscillators, affected by distributed delays in the coupling and we characterize the structure of the emanating solutions in the bifurcations, under the assumption that the delay kernels are equal to each other. The motivation comes from the fact that valuable quantitative and qualitative information about the occurrence and type of synchronous or partially synchronous solutions can be obtained from this linear analysis. We analyze stability of synchronized equilibria as a function of the parameters of a so-called shifted gamma-distributed delay, which allows to represent or approximate a large class of distributed delay kernels. We also present an asymptotic analysis method, which is particularly suitable for studying the effect of a distribution of delays on stability. We apply the methods to networks of coupled Lorenz systems, where we highlight that stability is favored by a distributed delay compared with a discrete delay with the same average value. Among others, we show that if the coupling is diffusive, the synchronized equilibria become asymptotically stable for large values of the coupling gain, i.e. the systems locally asymptotically synchronize at an equilibrium, independently of the network topology. Copyright (c) 0000 John Wiley \& Sons, Ltd.
\end{abstract}

Received ...

KEY WORDS: Nonlinear oscillators, gamma distributed delays, interconnected systems

\section{INTRODUCTION}

Systems of coupled nonlinear oscillators have been used to describe a variety of phenomena in different fields of science, ranging from electric network modeling to brain activity behavior. The emergence of synchronization in networks of oscillators with couplings given by various types of topologies has received much attention [6, 20, 23, 4]. The influence of discrete fixed delays on the consensus and synchronization of coupled subsystems is considered in $[18,12,16,19]$. However, as pointed out in several studies $[9,5,10,7]$, the behavior of interconnected systems is

${ }^{*}$ Correspondence to: Université de Lorraine, CRAN, UMR 7039 and CNRS, CRAN, UMR 7039, 2 av. Forêt de Haye, Vandœuvre-lès-Nancy, France Email: constantin.morarescu@univ-lorraine.fr

Copyright (C) 0000 John Wiley \& Sons, Ltd.

Prepared using rncauth.cls [Version: 2010/03/27 v2.00] 
often more accurately reflected by considering distributed delays affecting their interactions. Note in this context that fast varying delays in communication channels can be well approximated by distributed delays, whose kernel is fitted to the probability density function of the delay [13].

This paper considers the problem of relative stability in a network of nonlinear oscillators, namely the synchronization problem, where the coupling is diffusive and affected by distributed delays. Precisely we are performing a local stability analysis concerning the linearization of the system around synchronized equilibria. The aim is to characterize the occurrence of synchronous behavior as a function of the parameters of the distributed delay. Preliminary results have been reported in [15]. Here, we are going further by emphasizing the delay distribution effect on stability of the linearized system as well as the information we can get for the nonlinear one. We follow the approach of $[12,24]$, which is based on analyzing stability and bifurcations of synchronized equilibria. Even though the analysis is local it has been demonstrated in [24] that by combining the stability charts and the information on emanating solutions in bifurcations with a characterization of invariant manifolds, valuable qualitative and quantitative information about the occurrence and type of synchronous or partially synchronous motion can be obtained, even if the attractor under consideration is more complicated than an equilbrium (e.g., a chaotic attractor).

The contributions of the paper are two-fold. First, we will provide systematic tools for analyzing the stability and bifurcations of synchronized equilibria when the delays are modeled by a gammadistribution kernel with a gap. This tool is based on the linearized system around the corresponding synchronized equilibria. By an appropriate choice of parameters such a kernel allows to model a broad class of distributed delays [17]. The applicability will be demonstrated with a case-study on coupled Lorenz systems. Second, we will pay particular attention to analyzing the effect of a delay distribution on synchronized equilibria, in comparison to the case of a discrete ( point-wise) delay, where we use the computed stability crossing curves as well as the asymptotic methods of [13].

Intuitively the stabilizing effect of a distributed delay might be understood from the fact that, in contrast to a discrete delay, it acts as a low-pass filter, and, hence, it suppresses the propagation of high frequency signals which might otherwise be destabilizing. In [13] a systematic study of the stabilizing effect of fast varying delays on stability is investigated, based on a distributed delay comparison system obtained by averaging. In [21] distributed delays are used in modeling the driver's reaction in a car following system, and compared with existing models with discrete delay. In [1] it is illustrated that distributed delay facilitates amplitude death of coupled oscillators, meaning that stability islands in parameter spaces of equilibria merge in such a way that the oscillations disappear. At the end of this paper we will analyze a form of amplitude death in networks of coupled Lorenz systems, which exhibit chaotic behavior without coupling, but, for sufficiently large coupling gain, synchronize at an equilibrium. Finally, the potentially destabilizing effect of approximating distributed delays by discrete delays is important in developing robust prediction based controllers for systems with input and output delays, as it has been shown that approximating the distributed delays in the control law, which originate from the inclusion of finite impulse response (FIR) filters, by discrete delays using a numerical quadrature rule, might destabilize the control loop [25]. The instability mechanism, as well as remedies, are explained in, e.g., [14] and the references therein.

The structure of the paper is as follows. In Section 2 we describe the interconnected system and outline the main assumptions. In Section 3 we perform a linear stability analysis of synchronized 
equilibria as a function of the parameters of a shifted $\gamma$-distributed delay. In Section 4 we analyze the beneficial effect of distributed delays using an asymptotic method. Finally, in Section 5 the application to coupled Lorenz systems is considered.

\section{PROBLEM FORMULATION AND PRELIMINARIES}

We consider a set of $p$ identical oscillators coupled via a fixed, directed network topology. Each oscillator has a behavior described by the following nonlinear dynamics

$$
\dot{x}_{i}(t)=f\left(x_{i}(t)\right)+B u_{i}(t), y_{i}(t)=C x_{i}(t), i=1, \ldots, p
$$

where $x_{i} \in \mathbb{R}^{n_{x}}, \quad i=1, \ldots, p, \quad B, C^{\top} \in \mathbb{R}^{n_{x} \times m}$, and $f: \mathbb{R}^{n} \mapsto \mathbb{R}^{n}$ is twice continuously differentiable.

\section{Assumption 1}

We assume that for $u_{i}=0$ the system has at least one unstable equilibrium of focus type, i.e., the Jacobian has two complex conjugate eigenvalues in the open right half plane, which we denote by $x^{*}$ in what follows.

The network topology is described by the directed graph

$$
\mathcal{G}(V, E, \mathbb{G})
$$

characterized by the node set $V=\{1, \ldots, p\}$, a set of edges $E$ and a weighted adjacency matrix $\mathbb{G}$ defined by the entries $\alpha_{k, l}$. The edge $(k, l) \in E$ exists if and only if $\alpha_{k, l} \neq 0$. Moreover, we suppose that self-loops are not present in the graph, i.e. $\alpha_{l, l}=0, \forall l \in V$. Each vertex corresponds to an oscillator and the edges describe the coupling strength between them. We assume a diffusive coupling between the oscillators, where the information sent from other systems is affected by a distributed delay. More precisely, we couple the systems (1) by means of the control law

$$
u_{i}(t)=k\left(\sum_{l=1}^{p} \alpha_{i, l} \int_{0}^{\infty} g(\theta)\left(y_{l}(t-\theta)-y_{i}(t)\right) \mathrm{d} \theta\right), \quad i=1, \ldots, p,
$$

where $k>0$ represents the gain parameter and $g(\cdot)$ stands for the $\gamma$-distributed delay with a gap kernel given by

$$
g(\theta)= \begin{cases}0, & \theta<\tau \\ \frac{(\theta-\tau)^{n-1} \mathrm{e}^{-\frac{\theta-\tau}{T}}}{T^{n}(n-1) !}, & \theta \geq \tau\end{cases}
$$

where $n \in \mathbb{N}, T>0$ and $\tau \geq 0$. Note that $g(\theta) \geq 0$ for all $\theta \geq 0$ and $\int_{0}^{\infty} g(\theta) d \theta=1$. The average delay satisfies

$$
\tau_{\text {av }}:=\int_{0}^{\infty} g(\theta) \theta d \theta=\tau+n T .
$$

A motivation for the consideration of this type of delay distribution can be found in [17]. 
The coupled system (1)-(3) has an equilibrium

$$
\left(x^{*}, \ldots, x^{*}\right) \in \mathbb{R}^{(n, p)},
$$

which we denote the synchronized equilibrium.

\section{Assumption 2}

The graph $\mathcal{G}$ is strongly connected.

\section{Assumption 3}

The adjacency matrix $G$ is stochastic i.e. $\sum_{l=1}^{p} \alpha_{i, l}=1, \quad i=1, \ldots, p$.

The first assumption is natural in the context of synchronization while the second assumption will be motivated in what follows. The second assumption ensures that synchronous behavior is preserved if it characterizes the past, i.e., it guarantees the presence of a synchronization manifold.

We recall that Perron-Frobenius theorem for stochastic matrices assures that

1. $G$ has a simple eigenvalue equal to 1 and $[1, \ldots, 1]^{\top}$ is the corresponding right-eigenvector.

2. All eigenvalues of $G$ have modulus smaller than or equal to 1 .

In what follows we denote the eigenvalues of $G$ as $\lambda_{i}(G), i=1, \ldots, p$, where we take the following convention that $\lambda_{1}(G)=1$.

Following the coordinate transformation in [12], we can bring the system (1) and (3) in the form

$$
\begin{aligned}
& \dot{x}_{1}(t)=f\left(x_{1}(t)\right)+B k C\left(\sum_{l=1}^{p} \alpha_{1, l}\right) \int_{0}^{\infty} g(\theta)\left(x_{1}(t-\theta)-x_{1}(t)\right) \mathrm{d} \theta \\
& +B k C \sum_{l=1}^{p} \alpha_{1, l} \int_{0}^{\infty} g(\theta) e_{l}(t-\theta) \mathrm{d} \theta, \\
& {\left[\begin{array}{c}
\dot{e}_{2}(t) \\
\vdots \\
\dot{e}_{p}(t)
\end{array}\right]=\left[\begin{array}{c}
f\left(x_{1}(t)+e_{2}(t)\right)-f\left(x_{1}(t)\right) \\
\vdots \\
f\left(x_{1}(t)+e_{p}(t)\right)-f\left(x_{1}(t)\right)
\end{array}\right]-k\left(\left[\begin{array}{ccc}
\sum_{l=1}^{p} \alpha_{2, l} & & \\
& \ddots & \\
& & \sum_{l=1}^{p} \alpha_{p, l}
\end{array}\right] \otimes B C\right)\left[\begin{array}{c}
e_{2}(t) \\
\vdots \\
e_{p}(t)
\end{array}\right]} \\
& +k \tilde{G} \otimes B C\left[\begin{array}{c}
\int_{0}^{\infty} g(\theta) e_{2}(t-\theta) \mathrm{d} \theta \\
\vdots \\
\int_{0}^{\infty} g(\theta) e_{p}(t-\theta) \mathrm{d} \theta
\end{array}\right]-\left(k\left[\begin{array}{c}
\sum_{l=1}^{p} \alpha_{1, l}-\sum_{l=1}^{p} \alpha_{2, l} \\
\vdots \\
\sum_{l=1}^{p} \alpha_{1, l}-\sum_{l=1}^{p} \alpha_{p, l}
\end{array}\right] \otimes B C\right) \int_{0}^{\infty} g(\theta)\left(x_{1}(t-\theta)-x_{1}(t)\right) \mathrm{d} \theta,
\end{aligned}
$$

where $e_{i}=x_{i}-x_{1}, \quad i=2, \ldots, p$ and $\sigma(\tilde{G})=\sigma(G) \backslash\{1\}$. Assumption 3 guarantees the preservation of synchronized solutions (that is, $e_{2}(s)=\ldots=e_{p}(s)=0, \forall s \leq t$ implies $\dot{e}_{2}(t)=$ $\left.\ldots \dot{e}_{p}(t)=0\right)$. The forward invariant set, described by $x_{1}=\cdots=x_{p}$ is called the synchronization manifold. 
Note from (6) that the coupling is invasive, in the sense that the dynamics on the synchronization manifold are affected by the coupling. This property is inherent to diffusive coupling affected by transmission delays.

\section{SHIFTED $\gamma$-DISTRIBUTED DELAYS: STABILITY ANALYSIS OF SYNCHRONIZED EQUILIBRIA}

We perform a stability analysis of the equilibrium (5) in the parameter space $(k, T, \tau)$ and characterize the emanating solutions in the bifurcations.

Linearizing the system (1) and (3) around the synchronized equilibrium $\left(x^{*}, \ldots, x^{*}\right)$, we obtain

$$
\left[\begin{array}{c}
\dot{v}_{1}(t) \\
\vdots \\
\dot{v}_{p}(t)
\end{array}\right]=I \otimes(A-B k C)\left[\begin{array}{c}
v_{1}(t) \\
\vdots \\
v_{p}(t)
\end{array}\right]+k G \otimes B C\left[\begin{array}{c}
\int_{0}^{\infty} g(\theta) v_{1}(t-\theta) d \theta \\
\vdots \\
\int_{0}^{\infty} g(\theta) v_{p}(t-\theta) d \theta
\end{array}\right],
$$

where $A=\frac{\partial f}{\partial x}\left(x^{*}\right)$.

\subsection{Characteristic equation}

The characteristic function of (8) is given by

$$
\Delta(s, k, T, \tau)=\operatorname{det} F(s, k, T, \tau),
$$

where the characteristic matrix $F$ is defined by

$$
F(s, k, T, \tau)=I \otimes(s I-A+k B C)-G \otimes k B C \frac{e^{-s \tau}}{(1+s T)^{n}} .
$$

Using the factorization $G=P \Lambda P^{-1}$, where $\Lambda \in \mathbb{C}^{p \times p}$ is triangular and $P \in \mathbb{C}^{p \times p}$, the characteristic function may be decomposed as

$$
\Delta(s, k, T, \tau)=\prod_{i=1}^{p} \Delta_{i}(s, k, T, \tau)
$$

where

$$
\begin{aligned}
& \Delta_{i}(s, k, T, \tau)=\operatorname{det} F_{i}(s, k, T, \tau), \\
& F_{i}(s, k, T, \tau)=s I-A+k B C-k B C \lambda_{i}(G) \frac{e^{-s \tau}}{(1+s T)^{n}}, i=1, \ldots, p .
\end{aligned}
$$

\subsection{Eigenspaces and behavior on the onset of instability}

We investigate the eigenspace of the characteristic matrix (10), corresponding to a characteristic root. The presence of eigenvalues of $G$ of multiplicity larger than one, leads to discussions related to the dimension of the eigenspaces and significantly complexifies the characterization of solutions 
of the linearized system. For the sake of clarity and in order to keep the reader focused on the main objective of the section, we simplify the analysis by restricting ourselves to the generic case where all the eigenvalues of the adjacency matrix $G$ are simple. Let $E_{i}$ be the eigenvector of $G$ corresponding to the eigenvalue $\lambda_{i}(G), i=1, \ldots, p$.

If for some $l \in\{1, \ldots, p\}$, the equation $\Delta_{l}(s, k, T, \tau)=0$ has a simple root at $s=\hat{s}$ such that

$$
F_{l}(\hat{s}, k, T, \tau) V=0, \quad V \in \mathbb{C}^{n \times 1},
$$

then it can be verified that

$$
F(\hat{s}, k, T, \tau)\left(E_{l} \otimes V\right)=0 .
$$

This implies that the linearized system (8) has an exponential solution

$$
\left[\begin{array}{c}
v_{1}(t) \\
\vdots \\
v_{p}(t)
\end{array}\right]=c\left(E_{l} \otimes V\right) e^{\hat{\lambda} t}=c\left[\begin{array}{c}
E_{l, 1} V \\
\vdots \\
E_{l, p} V
\end{array}\right] e^{\hat{\lambda} t},
$$

with the constant $c$ depending on the initial conditions. In words, in an exponential solution of (8), corresponding to a zero of $\Delta_{l}(s, k, T, \tau)$, the relation between the state variables of an individual subsystem is determined by the null vector of $F_{l}$, while the relation between the corresponding state variables of the different subsystems is solely determined by the eigenvector $E_{l}$, corresponding to the $l$-th eigenvalue of the adjacency matrix $G$. This implies that all modes can be classified in at most $p$ types, based on the relations between the behavior of the different subsystems. Note for example that bifurcations of the synchronized equilibrium due to imaginary axis zeros of $f_{1}$ are bifurcations where the synchronized behavior is preserved in the emanating solution, as $E_{1}=\left[\begin{array}{llll}1 & 1 & \cdots & 1\end{array}\right]^{T}$.

In [24], for the case of discrete delay, the above information is used to predict the occurrence of synchronous or partially synchronous regimes as a function of parameters of the coupling, even if the attractor is different from an equilibrium. Thereby it complements the conventional, Lyapunov based analysis. To explain the main idea, let us assume that we have $p=4$ agents and that all unstable characteristic roots are due to zeros of $f_{1}$ and $f_{k}, k \in\{2,3,4\}$ such that $E_{k}=\left[\begin{array}{llll}1 & 1 & -1 & -1\end{array}\right]^{T}$. In addition, assume that the set $M:=\left\{x \in \mathbb{C}^{4 n_{x}}: x_{1}=x_{2} ; x_{3}=x_{4}\right\}$ is forward invariant with respect to the dynamics of the coupled system, i.e., it is a so-called partial synchronization manifold. From (15), we then conclude that close to the synchronized equilibrium the solutions are repelled but in such a way that the synchrony between agents 1 and 2, and the synchrony between agents 3 and 4 are maintained. This preservation of partial synchrony might be an indication (but no proof) that the manifold $M$ is stable, and the corresponding partially synchronous motion occurs.

See further Figure 2 and Figure 6. The special structure of the unstable eigenvectors and in particular the "Hopf 90" bifurcations in Figure 2 are an indication (but not more than that) of stable motion characterized by a 90 degrees phase shift. Such a behavior is effectively observed in Figure 6.

\subsection{Computation of stability crossing curves in parameter spaces}

Due to the specific form of the characteristic matrix (10), which can be pre-multiplied with $(1+s T)^{n}$, standard methods for the stability and bifurcation analysis of systems with discrete 
delays can be used. In Section 5 we will use the package DDE-BIFTOOL [3] in order to compute stability determining characteristic roots corresponding to the synchronized equilibria. Stability regions in the $(T, \tau)$ and $(k, \tau)$ parameter spaces will be computed by the package as Hopf bifurcation curves of the nonlinear coupled system. Here the computational cost can be significantly reduced by taking into account the factorization (11) of the characteristic matrix, as well as the following property.

\section{Proposition 1}

The following invariance properties of imaginary axis characteristic roots hold:

1. For $i \in\{1, \ldots, p\}$, the property $\Delta_{i}(j \omega, k, T, \tau)=0$ for $\omega>0$ implies that $\Delta_{i}\left(j \omega, k, T, \tau+\frac{2 \pi l}{\omega}\right)=0$, where $l=1,2, \ldots$

2. For $i, l \in\{1, \ldots, p\}$, such that $\left|\lambda_{i}(G)\right|=\left|\lambda_{l}(G)\right|$, and $\omega>0$, the equality $\Delta_{i}(j \omega, k, T, \tau)=0$ implies $\Delta_{l}(j \omega, k, T, \hat{\tau})$, where $\hat{\tau}=\tau+\frac{\angle\left(\lambda_{l}(G)\right)-\angle\left(\lambda_{i}(G)\right)}{\omega}$.

For computing stability crossing curves specifically in the $(T, \tau)$ parameter space, a semianalytical method can also be used, which extends the approach for single input-single output systems of [17] . More precisely, defining the frequency crossing set $\Omega$ as the set collection of all $\omega>0$ such that there exists a parameter pair $(T, \tau)$ satisfying $\Delta(j \omega, k, T, \tau)=0$, we have the following result.

\section{Proposition 2}

For a given gain $k^{*}$ and any $\omega \in \Omega$, all corresponding $(T, \tau)$ values satisfying $\Delta_{i}\left(j \omega, k^{*}, T, \tau\right)=0$ can be computed by

$$
\begin{gathered}
T=T_{l}=\frac{1}{\omega}\left(\left|z_{l}(\omega)\right|^{-2 / n}-1\right)^{1 / 2} \\
\tau=\tau_{l, m}=\frac{1}{\omega}\left(-\angle z_{l}(\omega)-n \arctan (\omega T)+2 m \pi\right), \\
m=0, \pm 1, \pm 2, \ldots
\end{gathered}
$$

where $z_{l}(\omega), l=1, \ldots, n_{\omega}$, satisfy $\left|z_{l}(\omega)\right|<1$ and are eigenvalues of the pencil

$$
\Lambda(z)=\left(-j \omega I+A-k^{*} B C\right)+z \lambda_{i}(G)\left(k^{*} B C\right) .
$$

Proof

Let us consider the following variable transformation

$$
z=\frac{e^{-j \omega \tau}}{(1+j \omega T)^{n}}
$$

Then, the equation $\Delta_{i}\left(j \omega, k^{*}, T, \tau\right)=0$ rewrites as

$$
\operatorname{det}\left(j \omega I-A+k B C-k B C \lambda_{i}(G) z\right)=0 .
$$

From the definition of $z$ it is clear that the admissible solution satisfy $|z|<1$. For such a solution $z_{l}$, the corresponding pair $(T, \tau)$ can be simply computed by (16) and (17). 
Proposition 2 gives rise to a frequency sweeping procedure for computing stability crossing curves in the $(T, \tau)$ parameter space, where as a function of $\omega$ the eigenvalues inside the unit disk of pencil $\Lambda(z)$ need to be computed. Note that, as the factor $\lambda_{i}(G)$ can be absorbed into $z$, only one eigenvalue computation per frequency is necessary. For a characterization of the crossing directions of imaginary axis roots, we refer to [17] as the results directly carry over.

The following result characterizes stability for large values of the gain parameter.

\section{Theorem 1}

Consider the coupled systems (1) and (3). Let $T>0$ be given. Assume that for $\tau=0$ the synchronized equilibrium is asymptotically stable for sufficiently large $k$. Then there is a constant $\hat{k}$ such that for every $k>\hat{k}$, the synchronized equilibrium is asymptotically stable independently of $\tau \geq 0$.

\section{Proof}

We show that for large values of $k$ eigenvalues cannot cross the imaginary axis when varying parameter $\tau$. The theorem then follows from the assumed stability for $\tau=0$.

The equation

$$
\Delta_{i}(j \omega, k, T, \tau)=0
$$

is equivalent to

$$
\operatorname{det}\left(I-(j \omega I-A+k B C)^{-1} k B C \lambda_{i}(G) \frac{e^{-j \omega \tau}}{(1+j \omega T)^{n}}\right)=0 .
$$

A necessary solvability condition is given by

$$
\rho\left((j \omega I-A+k B C)^{-1} k B C \frac{\lambda_{i}(G)}{(1+j \omega T)^{n}}\right)=1,
$$

with $\rho(\cdot)$ the spectral radius. This condition is always violated for large $k$. Indeed, in the complex plane the nonzero eigenvalues of the matrix

$$
\left(j \frac{\omega}{k} I-\frac{A}{k}+B C\right)^{-1} B C \lambda_{i}(G)
$$

converge to the curve

$$
\Omega \geq 0 \mapsto \frac{1}{j \Omega+1} \lambda_{i}(G)
$$

as $k \rightarrow \infty$, uniformly in the parameter $\omega \geq 0$. Furthermore, we have:

$$
\left|\frac{1}{1+j \Omega} \lambda_{i}(G)\right| \leq\left|\lambda_{i}(G)\right| \leq 1, \quad \forall \Omega \geq 0
$$

and

$$
\frac{1}{\left|(1+j \omega T)^{n}\right|}<1, \forall \omega>0 .
$$

It follows that zeros on the imaginary axis are not possible for large values of $k$. 


\section{ASYMPTOTIC ANALYSIS OF THE DELAY DISTRIBUTION EFFECT ON STABILITY}

In Section 3 we outlined how to compute stability information as a function of the parameters $n, T$ of the shifted $\gamma$-distributed delay. In this section we analyze the effect on stability of a transition from a discrete delay to a distributed delay. Here we use an asymptotic method originally proposed in [13] and later rediscovered in [2]. The obtained results are asymptotic, yet independent of the shape of the distributed delay.

The kernel of the distributed delay (4) can be expressed as

$$
g(\theta)=w\left(\theta-\tau_{\mathrm{av}}\right)
$$

where the function $w: \mathbb{R} \rightarrow \mathbb{R}$ satisfies the following properties,

$$
\begin{array}{ll}
w(\theta) \geq 0, \quad \forall \theta \in \mathbb{R}, \quad w(\theta)=0, \quad \forall \theta \leq-\tau_{\mathrm{av}}, \\
\int_{-\infty}^{\infty} w(\theta) d \theta=1, \quad \int_{-\infty}^{\infty} \theta w(\theta) d \theta=0 .
\end{array}
$$

The characteristic equation corresponding to the synchronized equilibrium can now be written as

$$
\Pi_{i=1}^{p} \operatorname{det}\left(s I-A+k B C-k B C \lambda_{i}(G) e^{-s \tau_{\text {av }}} \eta(s)\right)=0,
$$

where

$$
\eta(s)=\int_{-\tau_{\mathrm{av}}}^{\infty} w(\theta) e^{-s \theta} d \theta
$$

In this way we have, in the frequency domain description, separated the contribution of the delay in a factor $\exp \left(-s \tau_{\mathrm{av}}\right)$, related to the average delay, and a factor $\eta(s)$, related to distribution of the delay around this average value. Note that, specifically for the kernel (4), we have

$$
\begin{aligned}
& w(\theta)= \begin{cases}0, & \theta<-n T, \\
\frac{(\theta+n T)^{n-1} \mathrm{e}^{-\frac{\theta+n T}{T}}}{T^{n}(n-1) !}, & \theta \geq-n T,\end{cases} \\
& \eta(s)=\frac{\left.e^{n T s}\right)^{-s+s T)^{n}} \Rightarrow e^{-s \tau_{\mathrm{av}}} \eta(s)=\frac{e^{-s \tau}}{(1+s T)^{n}},}{}
\end{aligned}
$$

which is in agreement with the results in the previous section. Note further that the function $s \mapsto \eta(s)$ is entire and satisfies

$$
\eta(0)=1, \quad \eta^{\prime}(0)=0, \eta^{\prime \prime}(0)>0 .
$$

Let us now, instead of (4) and (19), consider the scaled kernel

$$
g_{\delta}(\theta)=\frac{1}{\delta} w\left(\frac{\theta-\tau_{a v}}{\delta}\right)
$$

where $\delta \in(0,1]$. In words, $\delta$ is a scaling factor describing the distribution of the delay around the average value. The smaller $\delta$, the more the delay is concentrated around the average value, and in the limit $\delta \rightarrow 0+$, function $g_{\delta}$ becomes a Dirac impulse, corresponding to a discrete delay $\tau_{\text {av }}$. This is clarified in Figure 1. 


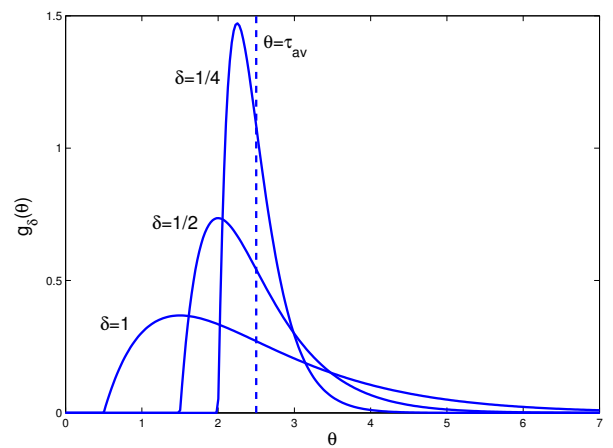

Figure 1. The scaled kernel $g_{\delta}$ for different values of $\delta$ and parameters $T=\tau=1, n=2$. For $\delta=1$ we have $g_{\delta}=g$. If $\delta$ is decreased the scaled kernel becomes more concentrated around $\tau_{\text {av }}=\tau+n T$. The limit $\delta \rightarrow 0$ corresponds to a Dirac impulse, associated with a discrete delay.

With the scaled kernel, the characteristic equation becomes

$$
h(s, \eta(s \delta))=0,
$$

where

$$
h(s, \eta(s \delta)):=\Pi_{i=1}^{p} \operatorname{det}\left(s I-A+k B C-k B C \lambda_{i}(G) e^{-s \tau_{\mathrm{av}}} \eta(s \delta)\right) .
$$

Consistent with the above observation on the limit $\delta \rightarrow 0+$, the contribution of the delay in (24) reduces to $e^{-s \tau_{\text {av }}}$ for $\delta=0$.

Consider an isolated characteristic root $s_{0}$ for $\delta=0$, i.e., for the case of discrete delay. From (24) we can compute the sensitivity of the root with respect to parameter $\delta$ at $\delta=0$. This sensitivity describes the initial effect on the root of distributing the delay around its original version. More precisely, by the implicit function theorem, Equation (24) describes a function $\delta \in\left[0, \delta_{m}\right] \mapsto r(\delta)$ for some $\delta_{m}>0$ satisfying $r(0)=s_{0}$ and $h(r(\delta), \eta(r(\delta) \delta))=0$ for all $\delta \in\left[0, \delta_{m}\right)$. An application of the chain rule, taking into account (21), yields

$$
\left\{\begin{aligned}
r^{\prime}(0) & =0, \\
r^{\prime \prime}(0) & =-\frac{\frac{\partial h}{\partial \eta}\left(s_{0}, 1\right)}{\frac{\partial h}{\partial s}\left(s_{0}, 1\right)} \eta^{\prime \prime}(0) s_{0}^{2} .
\end{aligned}\right.
$$

A corollary related to the stabilizing effect of a delay-distribution is the following, where we used $g^{\prime \prime}(0)>0$.

\section{Proposition 3}

Assume that for $\delta=0$, the rightmost characteristic roots of the synchronized equilibrium of the coupled system are simple and on the imaginary axis. Denote them by $j \omega_{i}, i=1, \ldots, m$. If

$$
\Re\left(\frac{\frac{\partial h}{\partial \eta}\left(j \omega_{i}, 1\right)}{\frac{\partial h}{\partial s}\left(j \omega_{i}, 1\right)}\right)<0 \quad i=1, \ldots, m,
$$


then the synchronized equilibrium is asymptotically stable for small values of $\delta$. If for some $i \in\{1, \ldots, m\}$ the inequality is reversed, the synchronized equilibrium is unstable for small values of $\delta$.

It is important to note that in the derivation of the above result only the properties (20) of $w$ are used, hence, it is largely independent of the way in which the delay is distributed. Finally, considering the behavior of a characteristic root $s_{0}$ as a function of $\delta$ as well as the nominal delay $\tau_{a v}$ around $\left(\delta, \tau_{\mathrm{av}}\right)=\left(0, \tau_{\mathrm{av}, 0}\right)$ leads to a root function $\left(\delta, \tau_{\mathrm{av}}\right) \mapsto r\left(\delta, \tau_{\mathrm{av}}\right)$. A straightforward computation leads to

$$
\Re\left(\frac{\partial^{2} r\left(0, \tau_{\mathrm{av}}\right)}{\partial \delta^{2}}\right)<0(>0) \Leftrightarrow \Im\left(\frac{\partial r\left(0, \tau_{\mathrm{av}}\right)}{\partial \tau_{\mathrm{av}}}\right)<0(>0),
$$

hence, the sensitivity of a root with respect to a distribution of the delay can be obtained as a byproduct from its sensitivity with respect to a change of size of the (average) delay.

In [13] the effect of fast time-varying discrete delays on stability of (stand-alone) control system was analyzed. In the first step the effect of a fast varying discrete delay and a distributed delay were correlated via an averaging based argument. In the second step the distributed delay was analyzed using the above asymptotic approach. The three examples in [13], including a model for a high speed cutting process, demonstrate the stabilizing effect of a fast variation and distribution of the delay. The qualitative properties of the spectrum of time-delay systems, described in Chapter 1 of [11], give an intuitive argument: in our intensive experiments with characteristic roots computations of delay equations it was often observed (but not always) that roots in the upper half plane follow the exponential envelope curves containing the spectrum and described by Proposition 1.10 of [11], that is, they cross the imaginary axis from left to right and with decreasing imaginary part. An example where this statement can actually be proven is the first order system $\dot{x}(t)=-k x(t-\tau), k>0$, based on the analytical computation of the crossing direction (using, e.g., the formulas of Chapter 4 of [11]). Example 2 in the article [8] on the asymptotic behavior of roots for large delay illustrates a much more complex scenario where multiple critical frequencies and crossings in both directions occur (see Figure 3.1 in the reference), characterized by decreasing imaginary part at the crossings as well. If this crossing behavior happens, a delay distribution has a stabilizing effect, by relation (26). In the next section we illustrate the stabilizing effect for networks of coupled Lorenz systems, where we obtain results independent of the network topology.

\section{CASE STUDY: COUPLED LORENZ SYSTEMS}

In this section the nonlinear oscillators (1) are specified as Lorenz systems:

$$
\begin{aligned}
& \left\{\begin{array}{l}
\dot{x}_{i, 1}(t)=\sigma\left(x_{i, 2}(t)-x_{i, 1}(t)\right), \\
\dot{x}_{i, 2}(t)=r x_{i, 1}(t)-x_{i, 2}(t)-x_{i, 1}(t) x_{i, 3}(t)+u_{i, 1}(t), \\
\dot{x}_{i, 3}(t)=-b x_{i, 3}(t)+x_{i, 1}(t) x_{i, 2}(t)+u_{i, 2}(t),
\end{array}\right. \\
& \left\{\begin{array}{l}
y_{i, 1}(t)=x_{i, 2}(t), \\
y_{i, 2}(t)=x_{i, 3}(t)-r, \quad i=1, \ldots p,
\end{array}\right.
\end{aligned}
$$


where $u_{i}=\left[u_{i, 1} u_{i, 2}\right]^{T}, y_{i}=\left[y_{i, 1} y_{i, 2}\right]^{T}$. The parameter values are given by

$$
\sigma=10, r=28, b=8 / 3 \text {. }
$$

Note that for $u_{i} \equiv 0$ each Lorenz system has three equilibria given by

$$
(0,0,0),( \pm \sqrt{b(r-1)}, \pm \sqrt{b(r-1)}, r-1)
$$

the latter two corresponding to unstable foci. Thus, Assumption 1 holds. Furthermore, with the parameter values (28) it exhibits a chaotic attractor [22].

If we linearize the coupled system (27) and (3) around the synchronized equilibrium

$$
\left(x^{*}, \ldots, x^{*}\right), \quad x^{*}=( \pm \sqrt{b(r-1)}, \pm \sqrt{b(r-1)}, r-1),
$$

then we obtain the linear system (8), where the matrices are specified as

$$
A=\left[\begin{array}{ccc}
-\sigma & \sigma & 0 \\
1 & 1 & \mp \sqrt{b(r-1)} \\
\pm \sqrt{b(r-1)} & \pm \sqrt{b(r-1)} & -b
\end{array}\right], \quad B=C^{\top}=\left[\begin{array}{ll}
0 & 0 \\
1 & 0 \\
0 & 1
\end{array}\right]
$$

It is easy to show that the stability of the linearized system does not depend on which equilibrium $x^{*}$ in (30) is considered, Therefore, we will restrict ourselves to the one in the positive octant.

In what follows we analyze the stability properties of the synchronized equilibria (30) in the $(k, T, \tau)$ parameter space. First we study the asymptotic behavior for large values of the gain parameters in $\$ 5.1$. For the standard parameters (28) this will allow us to make assertions about stability regions, stability switches and emerging behavior, which do not depend on the network topology. Next we present a numerical example in $\S 5.2$.

\subsection{Behavior for large gain values}

For $T=0$, i.e., the case of discrete delay, the behavior of the stability crossing curves for large gain values is described by the following result from [12].

Theorem 2

Consider a network of coupled Lorenz systems (27) with parameters (28) and coupling (3). Assume that $T=0$. Then there exists a number $\hat{k}>0$ and a function

$$
\tau^{*}:[\hat{k}, \infty] \rightarrow \mathbb{R}_{+}, k \mapsto \tau^{*}(k),
$$

satisfying the following properties:

1. there is a constant $\tilde{k}>\hat{k}$ such that for every $k>\tilde{k}$, the synchronized equilibrium has two characteristic roots in the open right half plane for all $\tau \in\left[0, \tau^{*}\right]$, while it is asymptotically stable for $\tau \in\left(\tau^{*}, \tau^{*}+\epsilon\right)$, with $\epsilon$ sufficiently small;

2. at $\tau=\tau^{*}$ Hopf bifurcation of the synchronized equilibrium of (27) and (3) occurs that is synchrony preserving (for decreasing delay); 
3. for all $k \in[\hat{k}, \infty]$ we can factor

$$
\tau^{*}(k)=\frac{v(k)}{k} \text { where } \lim _{k \rightarrow \infty} v(k)=0.586004 .
$$

Furthermore, the number $\hat{k}$ and the function (31) are independent of the number of subsystems and of the network topology.

In addition, in [12] asymptotic synchronization has been proven that for $k$ sufficiently large and $k \tau$ sufficiently small: boundedness of solutions proven using the Lyapunov functional

$$
V=\sum_{i=1}^{p} \gamma_{i}\left\{y_{i}(t)^{T} y_{i}(t)+k / 2 \sum_{i=1}^{p} y_{i}(\theta)^{T} y(\theta) d \theta\right\},
$$

with $\left[\gamma_{1} \ldots \gamma_{p}\right]$ the left eigenvector of $G$ corresponding to eigenvalue 1 , while stability of the synchronization manifold is inferred from a small gain argument. In fact, for $k$ large and $k \tau$ sufficiently small synchronized chaotic behavior is observed.

The following theorem shows that the qualitative behavior for large values of $k$ is significantly different if $T \neq 0$.

\section{Theorem 3}

Consider a network of coupled Lorenz systems (27) with parameters (28) and coupling (3). Assume that $T>0$. Then there is a constant $\hat{k}$ such that for every $k>\hat{k}$, the synchronized equilibrium is asymptotically stable independently of $\tau \geq 0$.

\section{Proof}

Given Theorem 1, it suffices to show that for $\tau=0$ all characteristic roots are in left half plane for sufficiently large $k$.

Let $i \in\{1, \ldots, p\}$. As $k \rightarrow \infty$ the function $\frac{\Delta_{i}(s, k, T, 0)}{k^{2}}=\operatorname{det} N_{i}(s, k)$, with

$$
N_{i}(s, k)=\left[\begin{array}{ccc}
s+\sigma & -\sigma & 0 \\
-\frac{1}{k} & \frac{s+1}{k}-\left(\frac{\lambda_{i}(G)}{(1+s T)^{n}}-1\right) & \frac{\sqrt{b(r-1)}}{k} \\
-\frac{\sqrt{b(r-1)}}{k} & -\frac{\sqrt{b(r-1)}}{k} & \frac{s+b}{k}-\left(\frac{\lambda_{i}(G)}{(1+s T)^{n}}-1\right)
\end{array}\right]
$$

uniformly converges on compact subsets of $\mathbb{C}$ to the function

$$
\left(1-\frac{\lambda_{i}(G)}{(1+s T)^{n}}\right)^{2}(s+\sigma)
$$

From Rouché's theorem it follows that, as $k \rightarrow \infty, 2 n+1$ zeros of $\Delta_{i}(s, k, T, 0)$ converge pairwise to the $2 n+1$ zeros of (33) (taking multiplicity into account). Similarly, letting $\tilde{s}=\frac{s}{k}$ we get 
$\frac{\Delta_{i}(\tilde{s} k, k, T, 0)}{k^{3}}=\operatorname{det}\left(\tilde{s} I-P_{i}(\tilde{s}, k)\right)$, where

$$
P_{i}(\tilde{s}, k)=\left[\begin{array}{ccc}
-\frac{\sigma}{k} & \frac{\sigma}{k} & 0 \\
\frac{1}{k} & -\frac{1}{k}+\left(\frac{\lambda_{i}(G)}{(1+\tilde{s} k T)^{n}}-1\right) & -\frac{\sqrt{b(r-1)}}{k} \\
\frac{\sqrt{b(r-1)}}{k} & \frac{\sqrt{b(r-1)}}{k} & -\frac{b}{k}+\left(\frac{\lambda_{i}(G)}{(1+\tilde{s} k T)^{n}}-1\right)
\end{array}\right] .
$$

Thus, $\frac{\Delta_{i}(\tilde{s} k, k, T, 0)}{k^{3}}$ uniformly converges on compact sets in the $\tilde{s}$-plane to $\tilde{s}(\tilde{s}+1)^{2}$. Rouché's theorem then implies that for sufficiently large $k$ the function $\Delta_{i}(s, k, T, 0)$ has two zeros equal to $k . \tilde{s}_{1,2}$, where

$$
\lim _{k \rightarrow \infty} \tilde{s}_{1,2}=-1,
$$

hence they move off the infinity without leaving the open left half plane.

The function $\Delta_{i}(s, k, T, 0)$ has exactly $2 n+3$ zeros, induced by $\operatorname{rank}(B C)=2$. We conclude from the previous observations that all these zeros are in the open left half plane for large $k$ if the zeros of (33) are in the open left half plane. This is the case for $i \in\{2, \ldots, p\}$. The case $i=1$ deserves special attention, since for $\lambda_{1}(G)=1$, (33) has two (rightmost) zeros equal to zero. The latter means that two zeros of $f_{1}(s, k, T, 0)$ approach the origin as $k \rightarrow \infty$. It remains to show that these approach the imaginary axis from the left.

With $\hat{s}=k s$ we define $g(\hat{s}, k):=f_{1}\left(\frac{\hat{s}}{k}, k, T, 0\right)$. By the expansion

$$
\left(\frac{1}{(1+\hat{s} T / k)^{n}}-1\right)=-\frac{\hat{s} T n}{k}+O\left(\left(\frac{\hat{s} T n}{k}\right)^{2}\right)
$$

and, again, an application of Rouché's theorem, it follows that, as $k \rightarrow \infty$, two zeros of $g(\cdot, k)$ converge to the finite eigenvalues of the pencil $-A+\hat{s}(T n) B C$. With the parameters (28) these eigenvalues are given by $\hat{s}_{1,2}=\frac{1}{T n}(-1.333 \pm 1.926 j)$. We conclude that the two zeros of $f_{1}(s, k, T, \tau)$ that converge to the origin as $k \rightarrow \infty$ satisfy

$$
s_{1,2}=\frac{1}{k} \hat{s}_{1,2}+O\left(\frac{1}{k^{2}}\right),
$$

hence, $\Re\left(s_{1,2}\right)<0$ for large $k$.

\subsection{Numerical examples}

We consider a ring topology with unidirectional coupling, described by the adjacency matrix

$$
G=\left[\begin{array}{ccccc}
0 & \cdots & & 0 & 1 \\
1 & 0 & & & \\
& \ddots & \ddots & & \\
& & & 1 & 0
\end{array}\right] \in \mathbb{R}^{p \times p}
$$


which has the following properties:

$$
\lambda_{l}(G)=e^{j \frac{2 \pi(l-1)}{p}}, E_{l}=\left[\begin{array}{c}
1 \\
e^{-j \frac{2 \pi(l-1)}{p}} \\
\vdots \\
e^{-j \frac{2 \pi(p-1)(l-1)}{p}}
\end{array}\right]
$$

for $l=1, \ldots, p$. If (13) is satisfied for $\hat{s}=j \omega, \omega>0$, then the emanating solution (15) becomes

$$
\left[\begin{array}{c}
\nu_{1}(t) \\
\vdots \\
\nu_{p}(t)
\end{array}\right]=c\left[\begin{array}{l}
V e^{j \omega t} \\
V e^{j \omega t-\frac{2 \pi(l-1)}{p}} \\
V e^{j \omega t-\frac{4 \pi(l-1)}{p}} \\
\vdots \\
V e^{j \omega t-\frac{2(p-1) \pi(l-1)}{p}}
\end{array}\right] .
$$

It can be interpreted as a traveling wave solution, where the agents follow each other with a phase shift of $360(l-1) / p$ degrees. Therefore, if the characteristic root $\hat{s}$ on the imaginary axis corresponds to a Hopf bifurcation of the original nonlinear system (27) and (3) for a critical value of some free parameter, we refer to this bifurcation as a "Hopf $360(l-1) / p$ " bifurcation.

For $p=4$ subsystems the stability regions in the $(k, \tau)$ parameter space are displayed in Figure 2, for $T=0$ (discrete delay). These results are in accordance with Theorem 2 . The blue curve segments correspond to the case where condition (25) is satisfied, the red segments to the case condition is not satisfied. We conclude that for $k>0.1$ the (initial) effect of a delay distribution is stabilizing.

In Figure 3 we depict the corresponding stability crossing curves for a shifted $\gamma$-distributed delay with parameters $T=0.01$ and $n=1$. The stability independent of $\tau$ for large $k$ is expected from Theorem 3. The similarity between the different curves is a consequence of the invariance properties in Proposition 1.

To relate the asymptotic results on the stabilizing effect of a delay distribution, indicated in Figure 2 by the colors, and the stability regions for the distributed delay with parameters $T=0.01$ and $n=1$, we superpose the stability regions shown in Figures 2 and 3 on Figure 4. It should be noted that in the horizontal axis we now display the average delay $\tau_{\mathrm{av}}=\tau+n T$.

In order to validate the above results and illustrate the information that can be obtained from the (local) analysis of synchronized equilibria (see also the discussion at the end of Section 3.2), we performed simulations of the nonlinear coupled system. First, we considered the case of discrete delays, $T=0$. We take $p=4, k=25$ and perform a simulation for the values of $\tau$ indicated in Figure 2 (left) with crosses, where the solution is initialized with a random perturbation of a synchronized equilibrium (the perturbation on each state variable is obtained from a normal distribution with zero mean and standard deviation one). For $\tau=0.008$ synchronized chaotic behavior is observed, see the top-left pane in Figure 5. This is consistent with the Hopf 0 bifurcation (synchrony preserved in the unstable manifold) and the asymptotic synchronization proven in [12]. For $\tau=0.05$ the stability of the synchronized equilbrium is confirmed (top-right pane of Figure 5). For $\tau=0.15$, depicted in the bottom pane of Figure 5, the behavior becomes quite interesting. The system exhibits a chaotic like attractor where the solutions of each subsystem are irregularly 

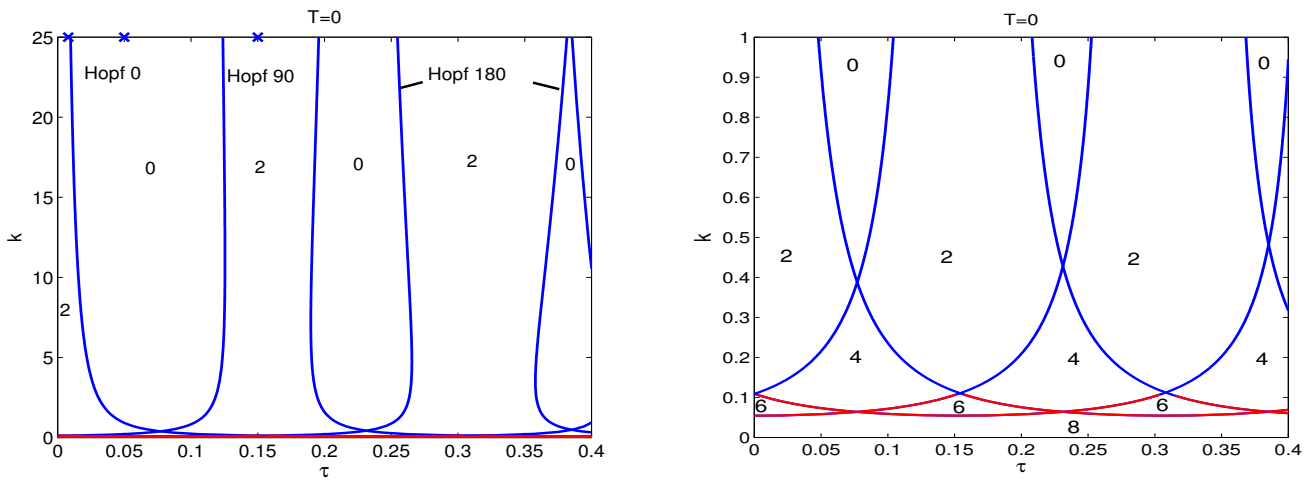

Figure 2. Stability regions of synchronized equilibria of 4 coupled Lorenz systems in a ring configuration, for $T=0$. The numbers refer to the number of characteristic roots in the right half plane. The Hopf bifurcation curves are classified based on type of emanating solution (35). The blue color indicates that condition (25) is satisfied. Whenever the blue curve bounds a stability region, the latter means that these values of $k$ and $\tau$ correspond to an asymptotically stable synchronized equilibrium for small $\delta>0$ (replacing the discrete delay by a distributed delay), i.e., the stability region grows locally.
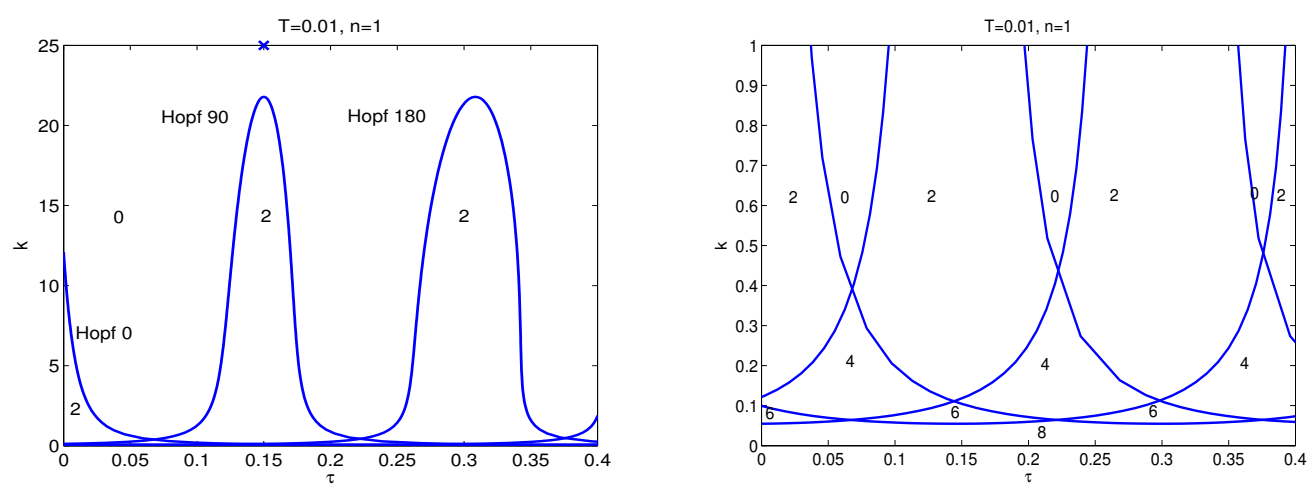

Figure 3. Stability regions of the synchronized equilibria of 4 coupled Lorenz systems in a ring configuration. Distributed delay with parameters $T=0.01$ and $n=1$.

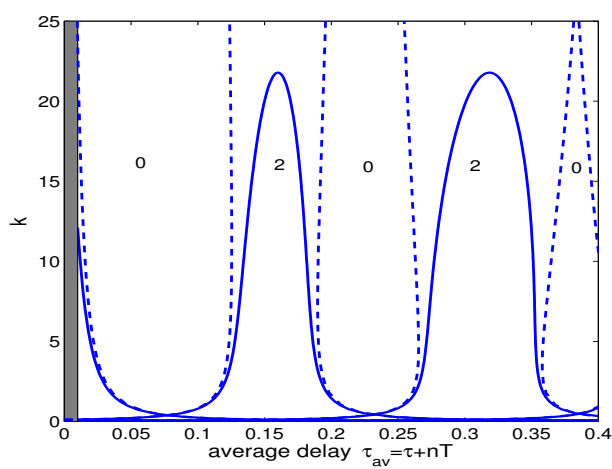

Figure 4. Stability regions of synchronized equilibria as a function of $k$ and $\tau_{\mathrm{av}}$, for $T=0$ (dashed curves) and $T=0.01, n=1$ (full curves). 

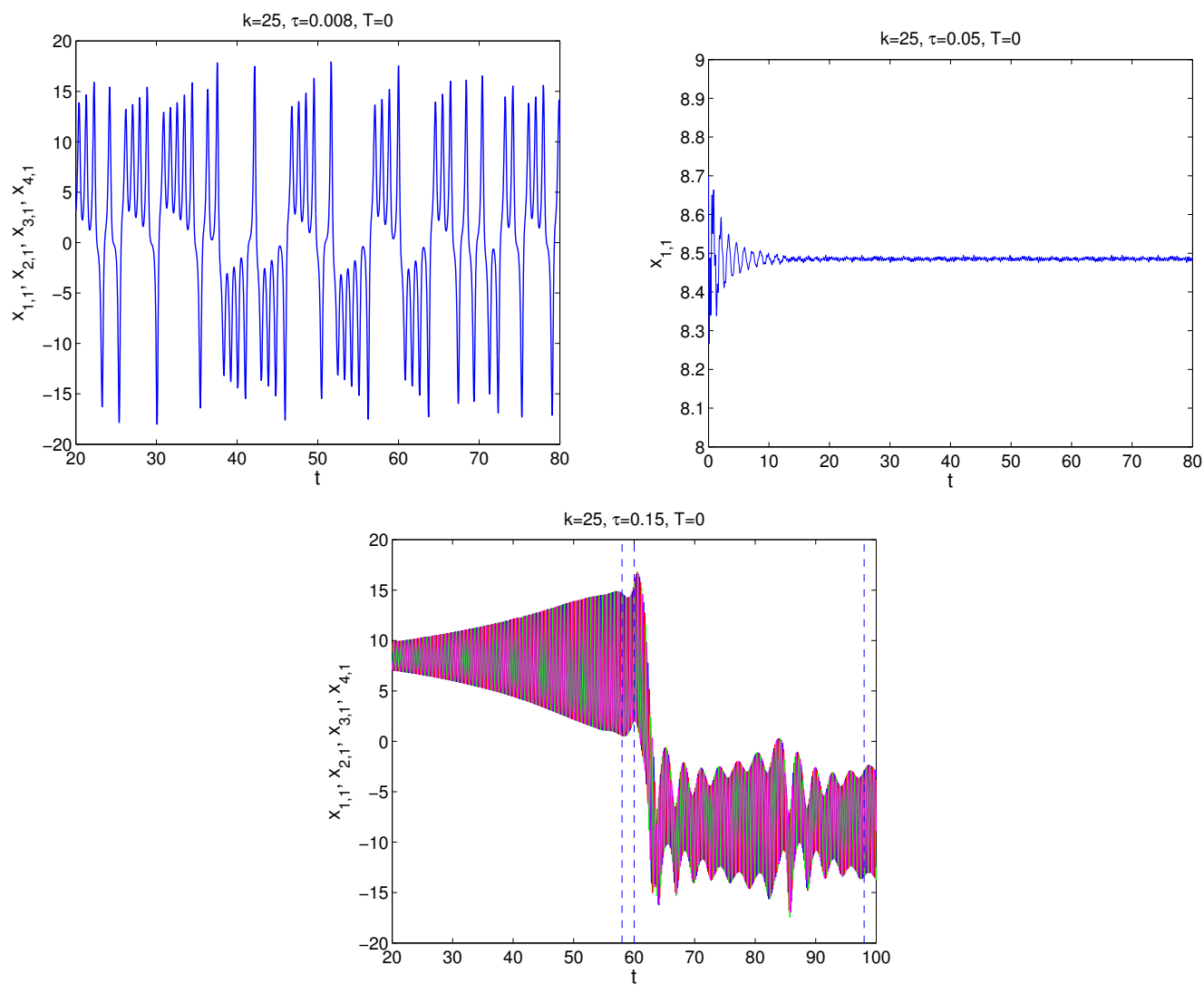

Figure 5. Simulations for 4 ring coupled systems, corresponding to parameter values indicated with crosses in Figure 2 (left). The delay values are $\tau=0.008$ (top-left), $\tau=0.05$ (top-right) and $\tau=0.15$ (bottom). In the latter case, a zoom on the time-windows indicated by the dashed lines is depicted in Figure 6.

swung from the neighborhood of one unstable equilibrium to the neighborhood of another unstable equilibrium, as for an uncoupled system. However, when zooming in on the solutions, it turns out that a phase shift of $\pi / 2$ degrees between the four systems is observed, see Figure 6 . This is in agreement with Figure 2 (left): the delay value $\tau$ lies in a region where the synchronized equilibria is unstable. The two surrounding Hopf 90 bifurcations curves imply that solutions are repelled from the synchronized equilibria, but with the property that the solutions in the unstable manifold are characterized by a $\pi / 2$ phase shift. Roughly speaking, solutions are the kicked away, but the kicks on the subsystems occur in a periodic, wavy manner. This illustrates that looking at the structure of eigenvectors at stability switches of synchronized equilibria may provide valuable information about the dynamics also beyond equilibria. Next, we considered the case of distributed delay with $n=1, T=0.01$ and $k=25$. Our simulations for different values of $\tau$ confirmed the delay-independent stability. See Figure 7 for the simulation for $\tau=0.15$ (indicated with a cross in the left pane of Figure 3).

Finally, Figure 8 shows the stability regions in the $(T, \tau)$ parameter space for $k=12$ and $k=25$. 

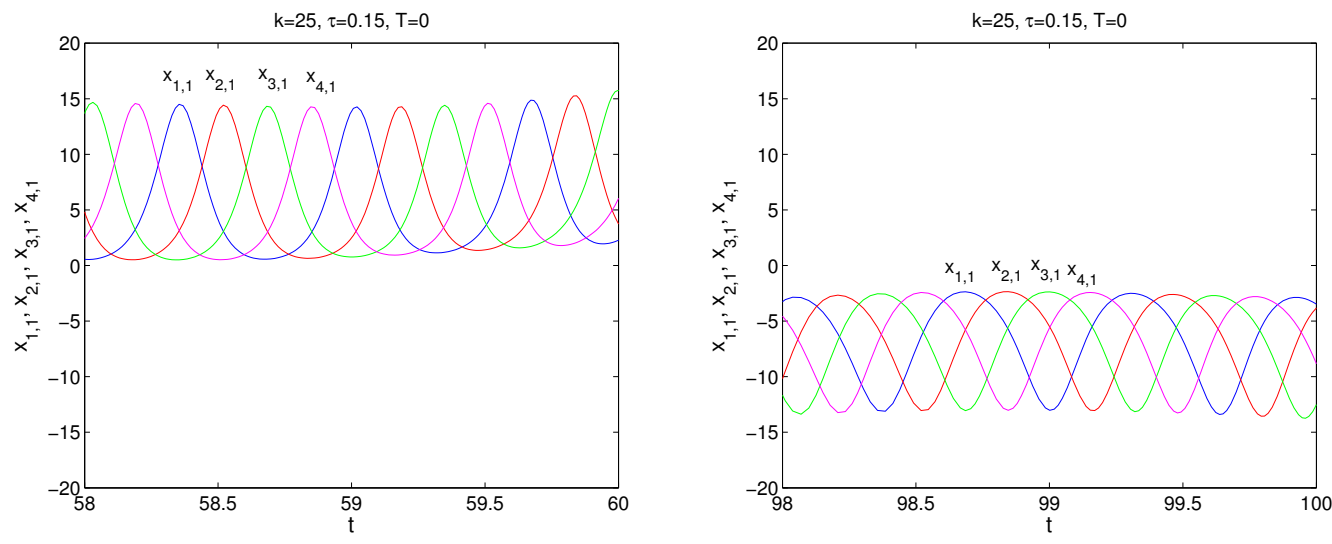

Figure 6. Simulations for 4 ring coupled systems. Zoom of Figure 5 (bottom) for the time-windows [58, 60] (left) and [98 100] (right). Notice the phase shifts of $\pi / 2$.

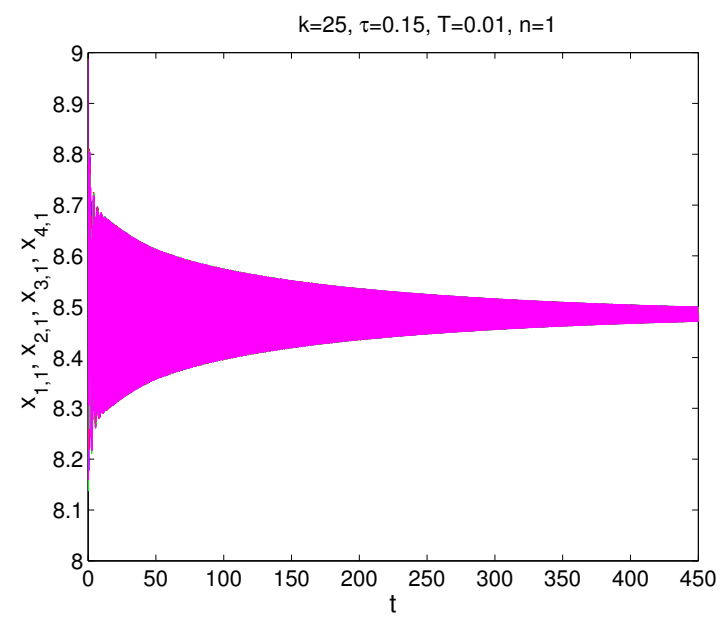

Figure 7. Simulation for 4 ring coupled systems, corresponding to parameter values indicated with a cross in Figure 3 (left). The delay values are $\tau=0.15, n=1, T=0.01$.

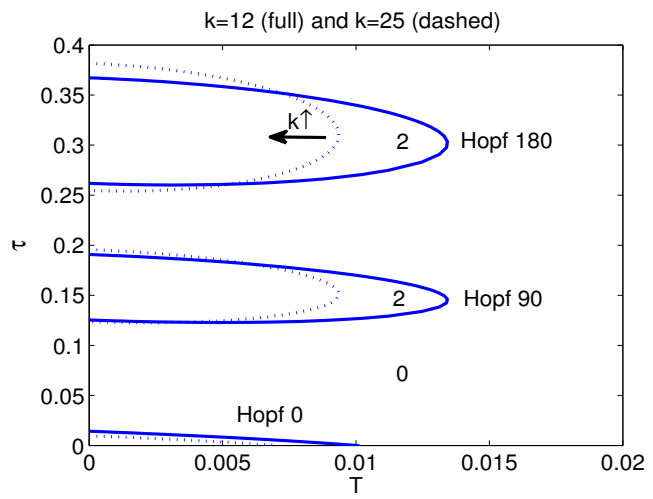

Figure 8. Stability regions in the $(T, \tau)$ parameter space for $k=12$ and $k=25$. 


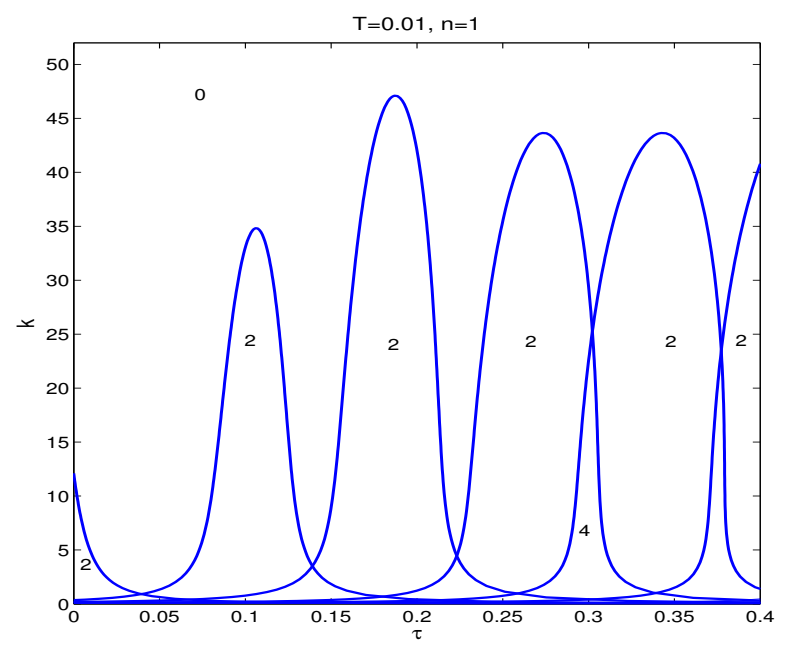

Figure 9. Stability regions of the synchronized equilibria of 8 Lorenz systems whose coupling is described by (36). Distributed delay with parameters $T=0.01$ and $n=1$.

In order to illustrate the applicability to other network configurations we consider a network of 8 Lorenz systems, where the communication graph is described by

$$
G=\left[\begin{array}{cccccccc}
0 & 0 & \frac{1}{2} & 0 & 0 & \frac{1}{2} & 0 & 0 \\
0 & 0 & 0 & 0 & 0 & 0 & 0 & 1 \\
\frac{1}{3} & \frac{1}{3} & 0 & 0 & 0 & 0 & \frac{1}{3} & 0 \\
\frac{1}{4} & \frac{1}{4} & \frac{1}{4} & 0 & 0 & 0 & \frac{1}{4} & 0 \\
\frac{1}{2} & 0 & 0 & \frac{1}{2} & 0 & 0 & 0 & 0 \\
0 & 0 & 0 & 0 & 0 & 0 & 1 & 0 \\
\frac{1}{4} & \frac{1}{4} & \frac{1}{4} & \frac{1}{4} & 0 & 0 & 0 & 0 \\
0 & 0 & 0 & 0 & 1 & 0 & 0 & 0
\end{array}\right],
$$

with eigenvalues

$$
\{1,0.2624 \pm 0.5678 j,-0.1676 \pm 0.4310 j,-0.4698 \pm 0.1681 j,-0.2500\}
$$

The coupling delay is described by a shifted $\gamma$-distributed delay. In Figure 9 we plot the stability regions of the synchronized equilibria as a function of the parameters $k$ and $\tau$ for $n=1$ and $T=0.01$. Notice, once more, the delay-independent stability for large values of $k$, a property independently of the network. Compared to Figure 3 the local maxima of the stability crossing curves are not equal to each other, which can be explained by the fact that not all eigenvalues (37) have equal magnitude (see also Proposition 1). 


\section{CONCLUSION}

We gained qualitative and quantitative insight in the effect of a delay distribution on relative stability in delay-coupled nonlinear oscillations, thereby modeling latency in the coupling by shifted $\gamma$ distributed delay. This was done by studying the stability regions of the synchronized equilibrium in the (coupling gain-delay) parameter space and by characterizing the structure of the emanating solutions in the bifurcations. In addition, we studied in a semi-analytical way, the effect of a transition from a discrete to a distributed delay, focusing on the effect on (relative) stability.

Applying the results to the particular case of coupled Lorenz systems we have shown that, independently of the network topology, for sufficiently large coupling gains the distribution of the delay has a stabilizing effect on the stability of the synchronized equilibrium. The fact that the synchronization mechanism is a high-gain mechanism, based on dominating the destabilizing terms in the synchronization error dynamics, suggests that a similar conclusion can be drawn for other types of nonlinear oscillators.

The quantitative tools for stability analysis can also be applied to the case where the coupling is described instead by

$$
u_{i}(t)=k\left(\sum_{l=1}^{p} \alpha_{i, l} \int_{0}^{\infty} g(\theta)\left(y_{l}(t-\theta)-y_{i}(t-\theta)\right) \mathrm{d} \theta\right), i=1, \ldots, p,
$$

however, the qualitative results are expected to be different. A major difference between (3) and (38) is that coupling (38) is non-invasive, in the sense that it vanishes on the synchronization manifold. As a consequence, the dynamics on this manifold are described by the dynamics of one uncoupled subsystem, and if the latter only has unstable equilibria, the synchronized equilibria of the network can never be stable.

\section{REFERENCES}

1. F.M. Atay. Distributed delays facilitate amplitude death of coupled oscillators. Physical Review Letters, 91:91:094101, 2003.

2. F.M. Atay. Delayed feedback control near hopf bifurcation. Discrete and Continuous Dynamical Systems - Series $S, 1: 197-205,2008$.

3. K. Engelborghs and D. Roose. Numerical bifurcation analysis of delay differential equations using DDEBIFTOOL. ACM Transactions on Mathematical Software, 28(1):1-21, 2002.

4. D. Gfeller and P. De Los Rios. Spectral coarse graining and synchronization in oscillator networks. Phys. Rev. Lett., 100:174104, 2008.

5. T. Insperger and G. Stépán. Semi-discretization of time-dely systems. Applied Mathematical Sciences. Springer, 2011.

6. Y. Kuramoto. Chemical Oscillations, Waves and Turbulence. Springer, New York, 1984.

7. Y.N. Kyrychko, K.B. Blyuss, and E. Schöll. Amplitude death in systems of coupled oscillators with distributeddelay coupling. European Physical Journal B, 84(2):307-315, 2011.

8. M. Lichtner, M. Wolfrum, and S. Yanchuk. The spectrum of delay differential equations with large delay. SIAM Journal on Mathematical Analysis, 43(2):788-802, 2011.

9. N. MacDonald. Biological delay systems: linear stability theory. Cambridge University Press, Cambridge, 1989.

10. W. Michiels, I.-C. Morărescu, and S.-I. Niculescu. Consensus problems with distributed delays, with application to traffic flow models. SIAM Journal on Control and Optimization, 48(1):77-101, 2009.

11. W. Michiels and S.-I. Niculescu. Stability and Stabilization of Time-Delay Systems: An Eigenvalue Based Approach. Advances in Design and Control 12. SIAM Publications, Philadelphia, 2007. 
12. W. Michiels and H. Nijmeijer. Synchronization of delay-coupled nonlinear oscillators: An approach based on the stability analysis of synchronized equilibria. Chaos, 19, 2009.

13. W. Michiels, V. Van Assche, and S.-I. Niculescu. Stabilization of time-delay systems with a controlled, timevarying delay and applications. IEEE Transactions on Automatic Control, 50(4):493-504, 2005.

14. S. Mondié and W. Michiels. Finite spectrum assignment of unstable time-delay systems with a safe implementation. IEEE Transactions on Automatic Control, 48(12):2207-2212, 2003.

15. I.-C. Morărescu, W. Michiels, and M. Jungers. Synchronization of coupled nonlinearoscillators with shifted gamma-distributed delays. In Proceedings of American Control Conference, 2013.

16. I.-C. Morărescu, S.-I. Niculescu, and A. Girard. Consensus with constrained convergence rate and time-delays. In IFAC Workshop on Time Delay Systems, TDS'10, 2010.

17. I.-C. Morărescu, S.-I. Niculescu, and K. Gu. Stability crossing curves of shifted gamma-distributed delay systems. SIAM Journal on Applied Dynamical Systems, 6(2):475-493, 2007.

18. T. Oguchi, H. Nijmeijer, and T. Yamamoto. Synchronization in networks of chaotic systems with time-delay coupling. Chaos, 18, 2009.

19. A. Panchuk, D.P. Rosin, P. Hövel, and E. Schöll. Synchronization of coupled neural oscillators with heterogeneous delays. International Journal of Bifurcation and Chaos, 23(12):1330039, 2013.

20. L. M. Pecora and T. L. Carroll. Master stability functions for synchronized coupled systems. Phys. Rev. Lett., 80(10):2109-2112, 1998.

21. R. Sipahi, F.M. Atay, and S.-I. Niculescu. Stability of traffic flow behavior with distributed delays modeling the memory effects of the drivers. SIAM Journal on Applied Dynamical Systems, 68(3):738-759, 2007.

22. S. H. Strogatz. Nonlinear dynamics and chaos: with applications to physics, biology, chemistry and engineering. Westview, Cambridge, 1994.

23. S. H. Strogatz. Sync: the emerging science of spontaneous order. Hyperion Press, 2003.

24. H.U. Unal and W. Michiels. Prediction of partial synchronization in delay-coupled nonlinear oscillators, with application to hindmarsh-rose neurons. Nonlinearity, 26:3101-3126, 2013.

25. V. Van Assche, M. Dambrine, J.-F. Lafay, and J.-P. Richard. Implementation of a distributed control law for a class of systems with delay. In Proceedings of the 3nd IFAC Workshop on Time Delay Systems, pages 266-271, Santa-Fe, USA, 2001. 